\title{
Digital Sunsensors, where there is no ease, in adding ten degrees
}

Johan Leijtens, Dick Broekmans, Frank Stelwagen

Johan Leijtens, Dick Broekmans, Frank Stelwagen, "Digital Sunsensors, where there is no ease, in adding ten degrees," Proc. SPIE 11852, International Conference on Space Optics - ICSO 2020, 118523F (11 June 2021); doi: 10.1117/12.2599546

SPIE Event: International Conference on Space Optics - ICSO 2021, 2021, Online Only 


\section{International Conference on Space Optics-ICSO 2020}

Virtual Conference

30 March-2 April 2021

Edited by Bruno Cugny, Zoran Sodnik, and Nikos Karafolas
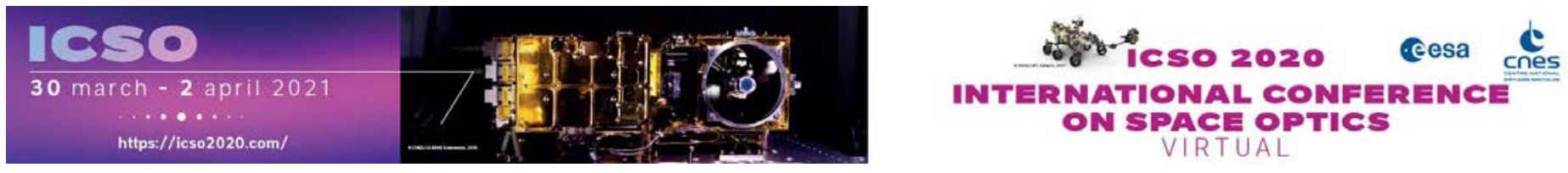

Digital Sunsensors, where there is no ease, in adding ten degrees

\section{Cesa isopmeatians ecnes}

International Conference on Space Optics - ICSO 2020, edited by Bruno Cugny, Zoran Sodnik, Nikos Karafolas, Proc. of SPIE Vol. 11852, 118523F - (c) 2021 ESA and CNES

CCC code: $0277-786 \mathrm{X} / 21 / \$ 21 \cdot$ doi: $10.1117 / 12.2599546$ 


\title{
Digital Sunsensors,
}

\section{Where there is no ease, in adding ten degrees.}

\author{
Johan Leijtens $^{1)}$, Dick Broekmans ${ }^{1)}$, Frank Stelwagen ${ }^{2)}$ \\ ${ }^{1)}$ Lens R\&D, 's-Gravendijckseweg 41b $2201 \mathrm{CZ}$ (the Netherlands) \\ Email: jls@lens-rnd.com tel. +31 (71) 2020123 \\ 2) SystematIC Design B.V., Elektronicaweg 20, 2628 XG Delft (the Netherlands)
}

\section{Summary}

Lens R\&D and SystematIC Design B.V. are currently involved, in frame of an Artes AT program, in designing a small true digital Sunsensor, intended to have an as large as possible Field Of View (FOV). Adding another 10 degrees to the common $60^{\circ}$ on axis field of view will even provide a redundant full spherical coverage with only 8 sensors, (instead of the required 10 sensors when a sensor with $60^{\circ} \mathrm{FOV}$ is used).

This conclusion prompted an investigation into the possibilities to increase the FOV to $69^{\circ}$ on axis.

During the following design considerations however, several optical issues were encountered, actually leading to a proposal to reduce the FOV to $62^{\circ}$ in diagonal ( $54^{\circ}$ on axis).

This paper focusses on these issues and effects, like polarisation dependent reflection, Sun spot spreading and shadowing effects of on-chip interconnects.

\section{Analogue versus digital Sunsensors}

Lens R\&D is currently specialized in high reliability analogue fine Sunsensors. The BiSon series Sunsensors are ESA ECSS qualified and radiation hardened sensors, capable of surviving the most stringent missions.

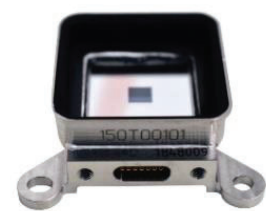

Figure 1 BiSon64-ET-B

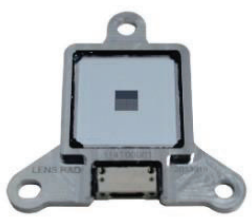

Figure 2 MAUS

Another Lens R\&D Sunsensor, the MAUS, is a low-profile derivative of the BiSon developments, specifically intended for CubeSat applications. As such, the MAUS is the first radiation-hardened CubeSat Sunsensor available on the commercial market. (The bare diodes used in the Lens R\&D Sunsensors have been successfully tested up to $8.10^{14} 1 \mathrm{MeV}$ electrons which is equivalent to $19.2 \mathrm{Mrad}$ TID and 2.5.10 ${ }^{10} \mathrm{MeV} . \mathrm{cm}^{2} / \mathrm{g}$ TNID) 
The Lens R\&D analogue fine Sunsensors are based on the use of custom developed radiation hardened four quadrant photodiodes and the use of a relatively thick Sapphire membrane $(650 \mu \mathrm{m})$, providing $1 \mathrm{~mm}$ Aluminium equivalent radiation shielding. Careful selection of the materials and custom developed glue ensure low outgassing and a very wide temperature range of operation. As a result, the sensors are extremely robust.

Lens R\&D analogue Sunsensors have a number of desirable properties like:

- They are small,

- Mechanically very robust

- Have a high radiation tolerance

- Are capable of a wide temperature range

- Are single-event upset and latch-up free

- And they are affordable

This leaves the question: why would anybody like to use something else than such a fine Analogue Sunsensor?

Well, there are essentially two reasons why a Digital Sunsensor will be preferred:

1. The digital data interface

2. Albedo insensitivity

These advantages of a Digital sensor will be addressed in the following paragraphs.

\subsection{A digital data interface}

There are several ways to provide for a digital interface, such as:

- Digitizing the analogue signals by using transimpedance amplifiers combined with a multiplexer and an

Analogue to Digital Converter (or multiple ADC's)

- Using a dedicated detector arrangement leading to a digital output (as for the Adcole digital Sunsensor) or

- Develop an Application Specific integrated Circuit (ASIC).

These solutions do solve the digital interface issue, but merely turn an analogue Sunsensor into an analogue Sunsensor with a digital interface. As a result, the sensors will still be hampered by significant measurement errors caused by albedo signals and reflections by spacecraft parts. In addition, also the cost associated with the interface electronics will in many cases supersede the cost of the bare sensor if a discrete solution is chosen.

One of the main advantages of using analogue Sunsensors is that the core sensors can be extremely robust. This is an important property as the sensors are always located on the outside of the satellite and as such directly exposed to the harsh space environment.

Depending on how well-designed the interface circuit is, the radiation tolerance can be quite high. When the interface electronics are located inside the spacecraft, the radiation tolerance will be increased by the shielding provided by spacecraft components. Integrating the interface electronics in the sensor itself, in the form of an ASIC, therefore requires careful consideration of the radiation environment and the lack of shielding by the satellite structure. If the sensor is not carefully optimised for radiation tolerance, this will lead to Single Event Upsets (SEU's) or even Single Event Latch-ups (SEL's), which are not only detrimental to the accuracy and availability of the sensors, but can even lead to destruction of the sensors.

In case of small sensors with low generated current levels there may however be no other option than to integrate a pre-amplifier or a more complicated form of data processing in the sensor itself. No level of processing on an analogue Sunsensor however will be able to avoid albedo errors, which brings us to the second reason to develop a true digital Sunsensor. 


\subsection{Albedo insensitivity with true Digital Sunsensors}

Analogue Sunsensors use either a single photodiode or four-quadrant photodiodes to determine the direction of the Sun. With a single diode, Coarse Sunsensors, this is done by looking at the cosine behaviour of the generated current as a function of the incidence angle. With the four-quadrant diodes, Fine Sunsensors, the ratio of the four generated currents is used. The main issue with both types of sensors however is the fact that they integrate all incoming light into a single output current per detector element. This leads to significant albedo induced errors, especially for low Earth orbiting satellites.

The intensity of the Sunlight is much higher than the intensity of light reflected by the Earth, but this reflected light will enter the sensor over a much wider opening angle. Where the opening angle of the Sun is approximately half a degree, the Earth can be visible over as much as 120 degrees when in low Earth orbit. Integrated over the full opening angle this leads to a significant albedo current. As this current cannot be separated from the current generated by the Sunlight, analogue Sunsensors will always be affected by Earth albedo signals. Similarly, reflections by spacecraft parts (be it specular or diffuse) will also lead to albedo errors.

Contrary to Star trackers, Sunsensors have no problems with albedo signals generated by the Moon. This is because the opening angle of the moon $\left(0.6^{\circ}\right)$ is almost the same as the opening angle of the Sun, but the reflection from the moon is semi-diffuse and the average albedo of the Moon is only 0.14 . Therefore, the Moon is actually relatively black and not generating signals that are of significance to Sunsensors.

Earth albedo signals can easily lead to errors in the order of $10^{\circ}$ or even in excess of $20^{\circ}$ for analogue Sunsensors, depending on opening angle orientation and orbit height. Even though these albedo signals only exist temporarily, they do lead to massive disturbances in the attitude control loop and significant software effort is required to mitigate the effects of these disturbances. Under rare circumstances, the use of wide FOV analogue Sunsensors has even led to a phenomenon that is called Earth lock, where the satellites attitude control system started to use the reflection from the Earth to control the orientation of the satellite rather than the position of the Sun. This can specifically happen when extensive ice cloud cover leads to a high average albedo signal.

Due to the large disturbances caused in the attitude control loops and the risk of Earth locking there is a strong preference to use true digital Sunsensors. A true digital Sunsensor uses a 2D array of sensing elements and a relatively small pinhole. As a result, the sensor divides the entire field of view in many smaller fields of view, each covering a smaller angular extend. By looking at the intensity within this smaller FOV, the sensor can discriminate between direct Sun illumination and illumination via a reflection on either a diffuse or reflective surface. Diffuse reflection will lead to a much lower signal because the light energy is spread over a much larger angular extend. Specular reflections can lead to signals in the same order of magnitude as those caused by direct Sun illumination but will only exist temporarily as the satellite is continuously moving around the Earth. Specular reflection will therefore only cause a "flash like" signal at detector level.

Splitting up the entire FOV in many smaller fields of view and by using thresholding the pixels illuminated by reflections can be simply distinguished from the sun illuminated pixels, leading to a highly reliable Sun detection and albedo insensitivity.

Even though various companies have tried to develop albedo insensitive Sunsensors, none of them have been able to develop a commercially successful product for high reliability applications. The available sensors were either too large and too expensive to be commercially successful (for instance the Galileo Avionica S3 or the JenaOptronik FSS) or not radiation tolerant enough (Newspace SS411 or Cube space Cubesense S).

Well documented attempts to make a single chip version by both Galileo Avionica and TNO have not led to a commercially available solution, and both attempts have been terminated to our knowledge. 


\section{The IBIS project}

After Galileo stopped with the ESA SSOAC (SunSensor On A Chip) activities, a new activity was started in frame of the advanced technology research program ARTES in cooperation with Lens R\&D. The intention of the program is to design a radiation tolerant and affordable albedo insensitive Sunsensor.

As affordability is concerned, a trade-off can only be performed at spacecraft level, otherwise the trade-off ends pretty quick with analogue Sunsensors being the best solution. Apart from affordability, a large number of aspects will have to be taken into consideration:

- Reliability

- Cost per sensor

- Number of sensors

- Interface requirements

- Performance of the solution

- Accommodation difficulties

- Processing software requirement

- Availability

- etc.

This paper doesn't claim to list all relevant aspects, nor do the authors claim in-depth knowledge of the relative importance of each of these, but a number of general observations can be made:

- The system reliability for a system using analogue Sunsensors is only marginally influenced by the actual sensor reliability and mainly determined by the processing electronics.

- The system costs are significantly influenced by the costs associated with the readout electronics

- Almost all interfaces to on-board computers are transforming to digital interfaces and thus avoiding analogue interfaces will de-complicate the design and increase flexibility

- Dedicated software processing to mitigate both Earth Albedo locking effects significantly complicates the use of analogue Sunsensors

- Cost and mass budgets associated with sensor wiring are significant.

- The number of reflections by spacecraft parts will increase with increasing FOV, not seldom leading to the need to exclude specific parts of the FOV by means of baffling if analogue Sunsensors are used

Reducing the number of sensors required for full spherical coverage will also lead to a reduction in the number of harnesses and interfaces. All of these reductions will lead to an overall cost and mass decrease and would favour the use of a sensor with a larger FOV.

In order to investigate the possibilities for increasing the sensors FOV, an in-depth investigation has been performed into the possibilities and potential drawbacks. The results of these investigations however have led to a number of interesting findings as described in the next paragraph. 


\section{Increasing the Field Of View}

The principal of operation of a true Digital Sunsensor is quite simple, as depicted in Figure 3:

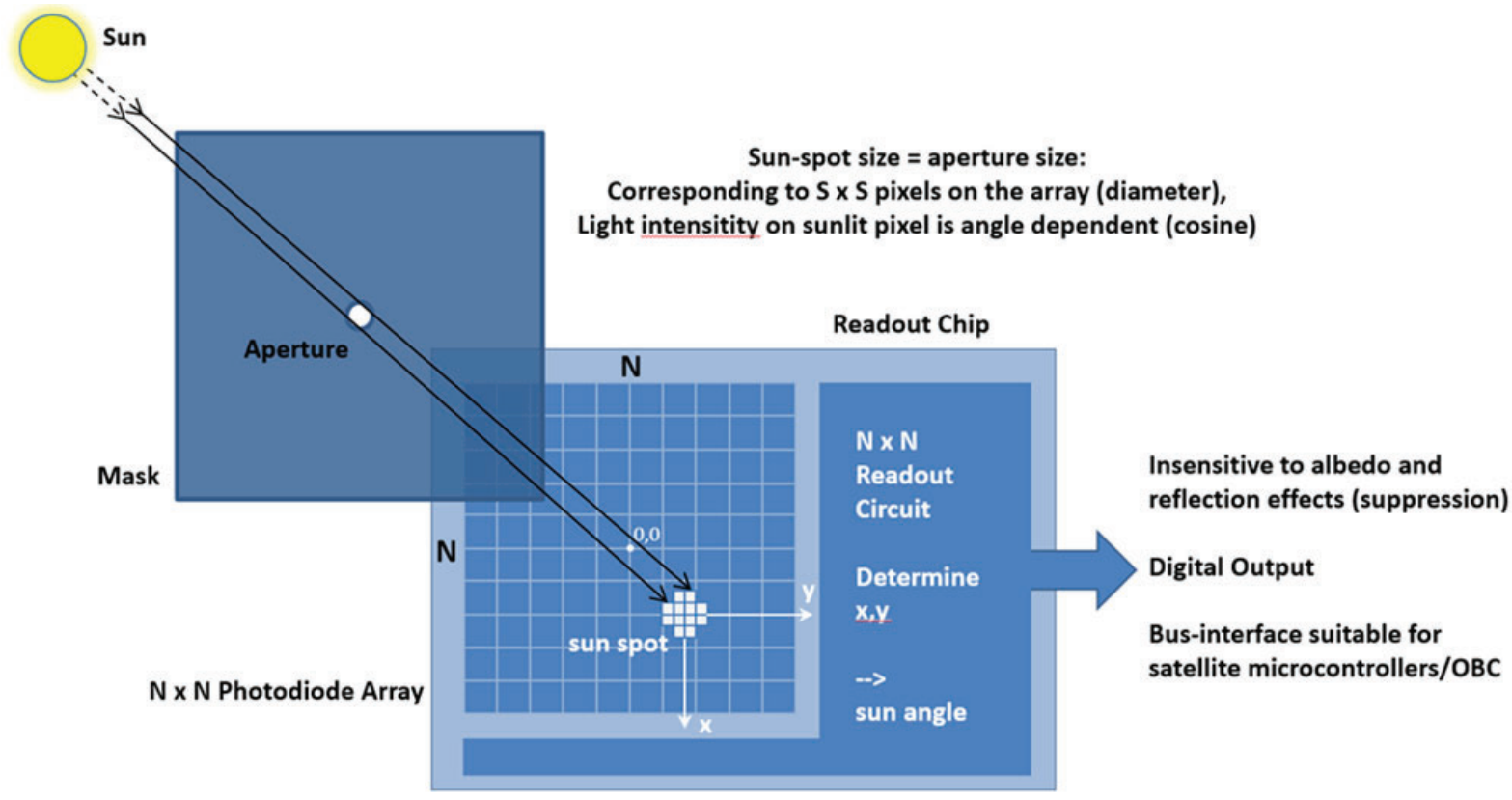

Figure 3 Digital Sunsensor principal diagram

The Sun illuminates a number of pixels in a photosensitive pixel array through a pinhole which is located at a certain distance from the detector surface as depicted in Figure 4.

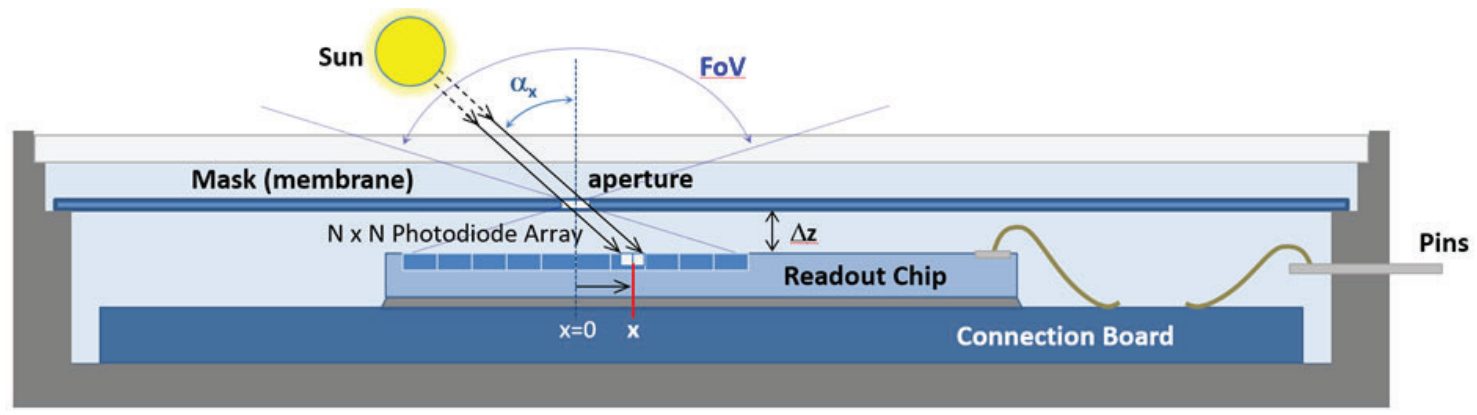

Figure 4 Digital Sunsensor principal build

In order to produce an affordable sensor, all active components of the chip must be integrated on a single readout chip to the largest extend possible, while external components are extremely costly. In addition, there are a number of other strong rationales to keep the sensors as small as possible:

- Lower material and component costs

- Lower mass for the same level of radiation shielding

- Lower energy absorption by Sun exposed surfaces 


\subsection{Low material and component costs}

The first criterion might be the least important one as the overall sensor costs will only be marginally influenced by the actual costs associated with the materials of the housing and standard components unless a number of active space qualified components are needed to build the sensor. As the number of sensors to be produced are expected to be limited, recoup of development costs and non-recurring engineering costs are expected to have a major influence on the overall cost of the sensors. As most of the functionality of the sensor is expected to be integrated in a single chip and the use of only passive support components is envisaged, the developments hold the promise to significantly reduce the overall costs associated with Sun sensing.

\subsection{Low mass solution}

Even though the actual component costs are expected to be limited, it is still expensive to launch items into space and mass budgets are still critical. Depending on the required level of radiation shielding, a significant wall thickness of the sensor will be required to meet the 15 or 18 years in GEO orbit radiation tolerance requirement.

Electric orbit raising is increasing in popularity but also has a significant drawback. During the electric orbit raising the Van Allen Belts are crossed on a regular basis, leading to a significant increase in collected radiation. As a result, a significantly higher level of shielding will be required for missions that use this orbit transfer method. The size and number of components in the cavity will determine the required cavity size. This cavity, plus the required level of shielding, will largely determine the total mass of the system.

It should be realized that radiation shielding is related to the specific mass of the applied material and that using materials providing higher levels of radiation shielding will decrease the wall thickness but will not decrease the mass due to the higher density of the material. Consequently, the only way to keep the mass low is to keep the sensors small.

\subsection{Low power absorption and dissipation}

In addition to the above, it should be realized that a larger sensor will lead to a larger Sun-exposed area. For ease of operation, it is strongly preferred if the use of the sensors doesn't require the application of any multi-layer isolation material to keep the sensor from being heated by the incident Sun light. As the surface area quadratically increases with both the length and the width of the cavity, the absorbed energy quickly increases with increasing sensor size. As the units are intended to be conductively controlled to keep the temperature within operational limits, the added absorption means increased temperatures and temperature differences as well as potentially the need for increased thermal conductance of the mechanical interface.

Larger sensors can easily absorb several Watts. This should be easily recognized as a strong rational to keep the sensors as small as possible to avoid thermal issues. The majority of the absorbed heat together with the power dissipated by the electronics will have to be rejected through the mounting feet of the sensors in case the unit is to be conductively controlled. Therefore, a small surface and low power dissipation are definitively high on the priorities list for the new development.

\subsection{Wide Field Of View}

While looking at Figure 4, it can be seen that one of the critical measures in a highly integrated digital Sunsensor is the height of the membrane above the sensing surface. This height (together with the physical size of the detectors sensor part) will determine the sensors FOV. By reducing this height, the same sensor will exhibit a larger FOV, but there are a number of effects which put a limit on this. With al FOV larger than $75^{\circ}$ in diagonal even full spherical FOV coverage with less sensors can be achieved. Therefore, a larger FOV is preferred, as long as the albedo sensitivity is not negatively affected. Consequently, an investigation was started into the FOV limiting factors. 


\subsubsection{Type of wirebond}

The minimum distance between the sensors light sensitive surface and the pinhole on the membrane is limited by the height of the wirebonds, including safety margins, while it must be avoided that the wirebonds touch the membrane. Wedge/wedge type wirebonds have a very low profile but need a larger distance between the chip edge and the contact point for the lower wedge, thus leading to larger packages. In addition, the extending part of the wedge will protrude over the chip and might obscure part of the active area, thus leading to dead spots. One of the most important aspects though is the fact that a very small distance between the sensor and the membrane will lead to a very high variation in the sensor's FOV due to for instance thickness variations in the glue and tolerances on the housing and ceramic thickness.

Ball/wedge bonds lead to a practical height limitation of $1 \mathrm{~mm}$ between the membrane and the sensor surface. This bonding method requires a much smaller area around the chip, thus limiting the size of the package. As a result, the use of ball/wedge bonds is preferred.

\subsubsection{Chip size}

The digital Sunsensor is implemented as a single chip device. Preliminary designs show that the chip size is mainly determined by the size of the sensor array. Presuming that the number of pixels in the array remain constant, the size of the processing electronics will be constant, but at a constant height of the membrane, the chip will have to be significantly larger as shown in below Figure 5 .

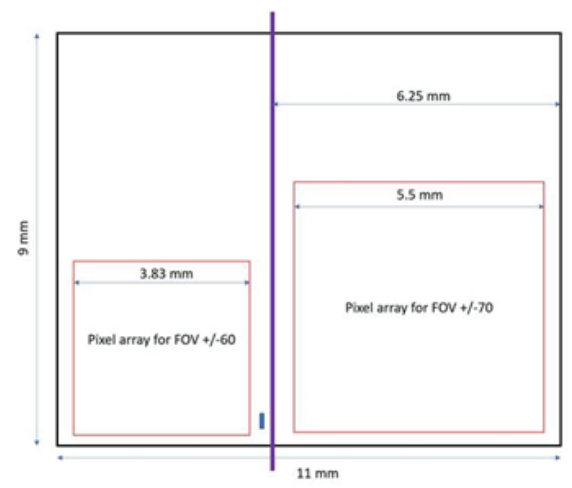

Figure 5 chip size for $60^{\circ}$ and $70^{\circ}$ FoV design

The $70^{\circ}$ design has approximately twice the size of the $60^{\circ}$ design, leading to a larger overall sensor, less chips per wafer and a lower production yield. Therefore, from a cost, size and yield point of view, the $60^{\circ}$ design is preferred.

\subsubsection{Negative effects and unwanted variations on Signal level}

One of the main concerns while designing a digital Sunsensor is the expected signal level. Basically, a digital Sunsensor determines the Sun by looking at the pixels for which a certain intensity threshold is exceeded. This will automatically discriminate between direct Sun inputs and inputs created by reflections (Earth Albedo) and leads to the so much desired albedo insensitivity.

\section{The Cosine Effect}

Increasing the FOV however has a number of negative effects on the intensity variations experienced by the pixels. The first prominent intensity variation is related to the cosine of the angle of incidence. This effect is barely noticeable around Zenith, but already reduces the intensity to half of the Zenith intensity at the end of the field of view as can be seen in Figure 6. 


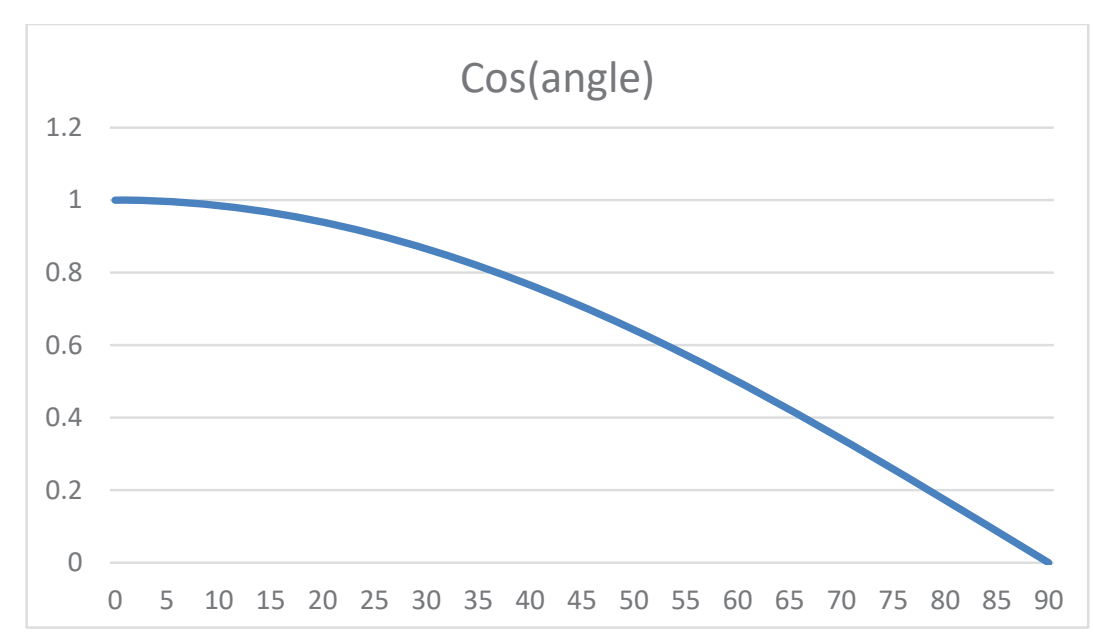

Figure 6 cosine

Where a reliable Sun detection will require an intensity compensation of a factor of 2 for angles up to $60^{\circ}$, extending to $70^{\circ} \mathrm{FOV}$ already requires a factor of 3 compensation. Whereas the FOV in diagonal will extend significantly beyond the FOV on axis, a $75^{\circ} \mathrm{FOV}$ in diagonal leads to a FOV less than $70^{\circ}$ on axis but will require an intensity compensation by already a factor 4 . Such a wide intensity variation is far more difficult to compensate than the factor of 2 associated with a $60^{\circ} \mathrm{FOV}$.

The cosine effect on the intensity however is not the only effect relevant for the intensity of the generated signal. The spot size variation as a result of the Sun's opening angle is another issue of concern for very large FOV's.

\section{The Twilight Effect}

The Sunlight is generally considered to be near parallel. However, due to the size and distance of the Sun, the light has an opening angle of approximately half a degree though. This leads to certain pixels being in what is dubbed "the twilight zone".

In order to explain this phenomenon, one has to look at Figure 7. The light from the Sun is entering the sensor through a membrane (on the left) that has a translucent pinhole with a diameter $\mathrm{D}$. This will project a spot with an exact diameter of $\mathrm{D}$ on the chip (as depicted on the right) if the beam would be $100 \%$ parallel. But, while the sun has an opening angle of $0.5^{\circ}(\omega)$, this will lead to a bigger and partly overlapping area of the sensor receiving light with different intensity $(\Delta \mathrm{D})$ This area will grow with increasing input angles, whereas the core size of the image that receives full illumination remains the same. It should be realized however that the core intensity already decreases with the cosine of the input angle as previously discussed and that the twilight pixels will have a decreased intensity the further they move from the side of the image spot. 


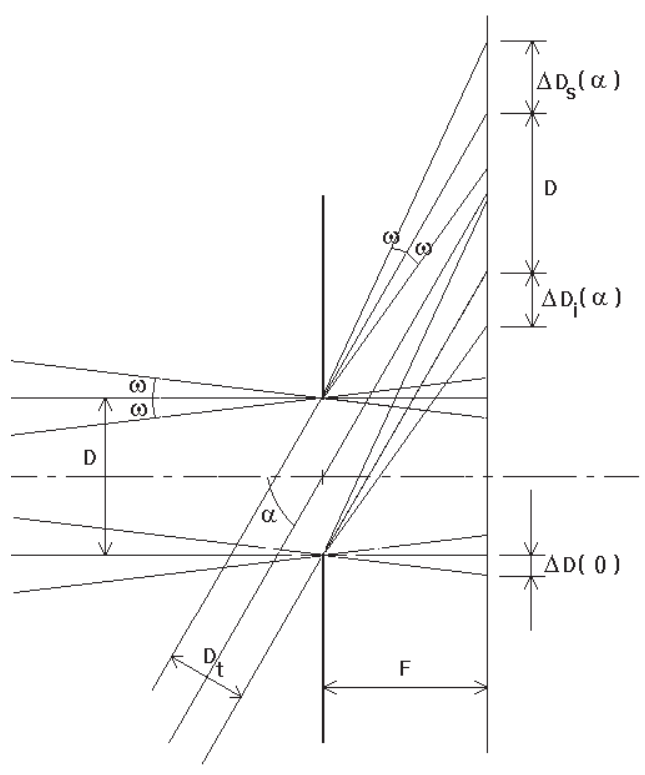

Figure 7 twilight zone pixels

The current design of the sensor is based on a configuration where the pinhole has a nominal size of ten pixels and each pixel represents a $0.5^{\circ}$ opening angle. Under these conditions, a fairly straight forward calculation can be made to show how many pixels are influenced by the opening angle of the incoming radiation. The result of this calculation is shown in Figure 8.

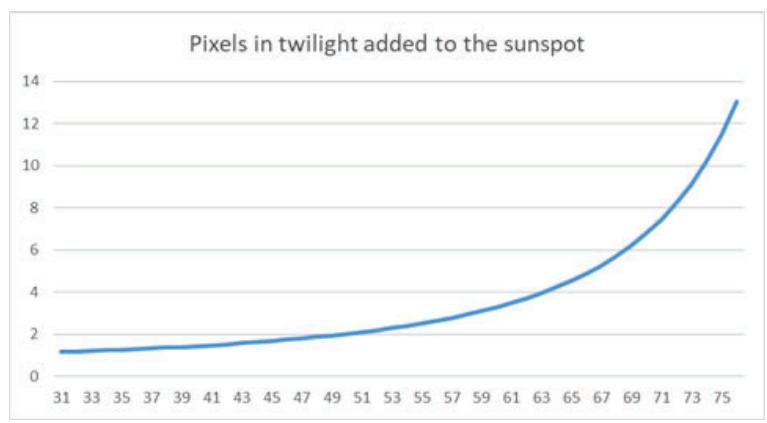

Figure 8 Twilight zone pixels

As can be seen, the number of pixels is relatively constant up to some $50^{\circ}$ but then starts to increase tangentially. The increase in number of twilight zone pixels is such that for angles larger than $65^{\circ}$ there are no core pixels left that are illuminated with the full beam intensity. This would further complicate a reliable detection leading to the need to increase the size of the pinhole (in turn leading to a larger pixel array) or a more complicated intensity compensation.

\section{Polarization effects}

Another phenomenon that needs to be taken into account when calculating the expected signal levels is the polarization dependent behaviour. As the light passes from vacuum into the silicon detector a significant polarization effect exists as a function of the angle of incidence and the polarisation state of the light, as shown in Figure 9. 


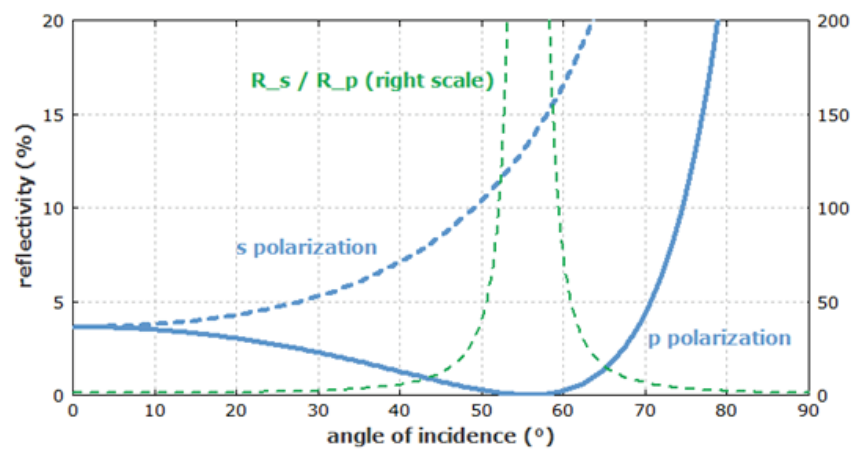

Figure 9 polarization dependent reflection

Where the reflection at normal incidence is calculated to be $4 \%$ (determined by the difference in refraction coefficient), the reflectivity for S and P polarized light is strongly dependent on the polarization state of the light.

If we look at the signal loss due to the reflection on the vacuum to silica interface it becomes obvious that this has a major impact for angles larger than 60 degrees. The graph in Figure 10 not only shows the reflection losses as a function of input angle, but also tabulates the extra signal loss over nominal incidence signal loss.

The $4 \%$ nominal signal loss can be contributed for in the calculations and even the $9 \%$ at the $60^{\circ}$ input angle could be accounted for (leading to a $\pm 5 \%$ intensity variation). The $25 \%$ signal loss for input angles of $75^{\circ}$ however are deemed problematic, especially because the exact configuration of the lower layers is not known and the reflection on the lower $\mathrm{SiO} 2$ to $\mathrm{Si}$ interface will also show the polarization dependent reflection, thus reflecting even more of the $\mathrm{S}$ polarized light. This transition will lead to another $15.5 \%$ reflection and signal loss at nominal incidence, but this signal loss is as before taken into account during the definition of the quantum efficiency. Nevertheless, it is strongly advised to try and stay below the Brewster angle of the vacuum to $\mathrm{SiO} 2$ interface as much as possible (which is $56^{\circ}$ ) as above this angle the reflection will strongly increase.

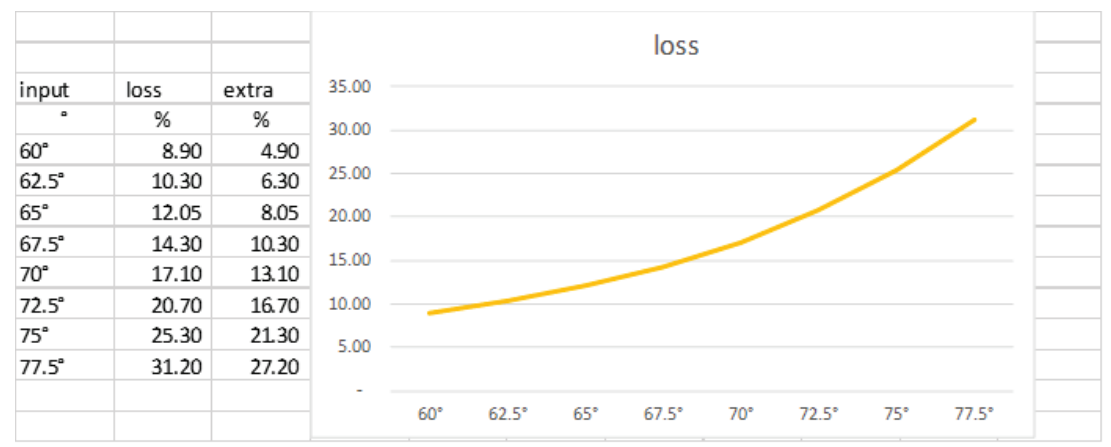

Figure 10 calculated extra polarization loss

\section{Track Shadowing}

Despite the fact that the tracks of the integrated circuit are located very close to the actual junction, for small pixels (as used in the digital Sunsensor), the shadowing effect can play an important role if not properly considered. The basis of this phenomenon is shown in Figure 11. This figure shows the metallization stack as defined by the supplier of the CMOS chips. 


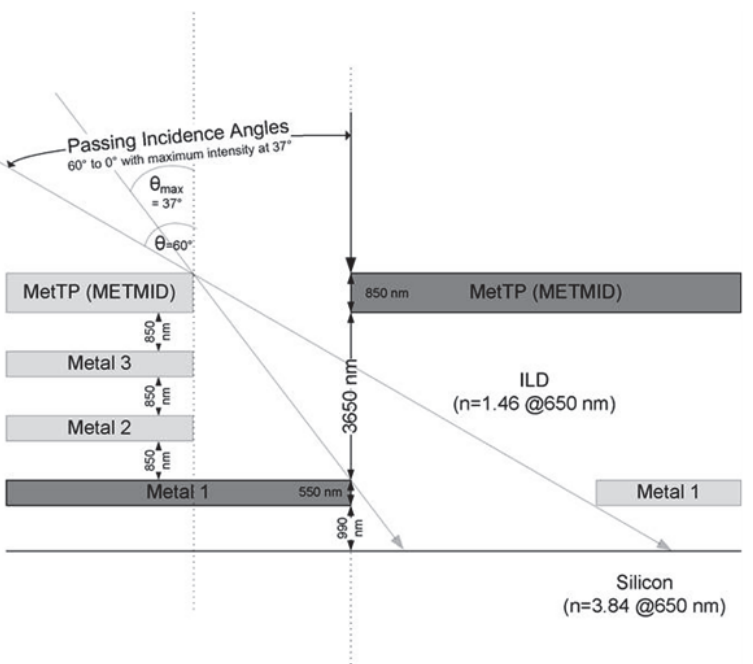

Figure 11 track shadowing

The separating material between the tracks (forming the optical window) is Silicon dioxide. The thickness of these separating layers is given in Figure 11, but are close to $1 \mu \mathrm{m}$ for each of the layers. This means that using a four-layer metal stack, the total stack thickness (excluding the covering layer) can be calculated to be around $6 \mu \mathrm{m})$. Due to the higher refractive index of the silica, the optical angle will be reduced from $60^{\circ}$ to some $37^{\circ}$, but this still means that the spot will hit the actual silicon not closer than $3 \mu \mathrm{m}$ from the edge of the upper metal layer. As the current design anticipates to use $10 \mu \mathrm{m}$ pixels it is obvious that this will have a detrimental effect on the apparent quantum efficiency and a significant amount of signal will be lost unless specific design measures are taken. As the effect grows with the tangent of the angle, it will worsen drastically with increasing input angles.

This is the final reason identified why it seems ill advised to try and make a digital Sunsensor with a FOV larger than $60^{\circ}$ in diagonal.

\section{Adding things up}

\section{There is no ease, in adding ten degrees}

The main reason to increase the Field Of View would be to allow covering a full spherical field of view with 8 instead of 10 sensors while providing single sensor failure redundancy. Covering the full spherical FOV with six sensors requires a FOV of $60^{\circ}$ in diagonal. This can be calculated to be equivalent to $54^{\circ}$ on axis. Such a sensor can also be used to provide single failure redundant coverage with 10 sensors.

As a smaller FOV significantly reduces signal variations and implementation difficulties, it is proposed to reduce the FOV to slightly over $60^{\circ}$ in diagonal so as to have some overlap but refrain from increasing the field of view. 


\section{Conclusions.}

Some background information has been given about the rationales for the development of a small and highly reliable Digital Sunsensor. Furthermore, the paper elaborated on various rationales related to the selection of the final FOV for the sensor.

The sensor should be (in order of importance):

- Highly reliable

- Affordable

- Albedo insensitive

- Equipped with a digital data interface

- Conductively cooled

- Low weight

Given the need for a highly reliable Sun detection, it seems ill advised to increase the FOV much beyond $60^{\circ}$ in diagonal. Doing so would require to lower the detection threshold, thus increasing Albedo sensitivity and false Sun detection probability or a complicated angle dependant detection threshold. The latter will be difficult to implement and verify, thus increasing development risk and system complexity and decreasing reliability. Decreasing the FOV below $60^{\circ}$ in diagonal would require more sensors to get full spherical coverage, in turn decreasing the affordability of the solution. Therefore, a guaranteed FOV of slightly more than $60^{\circ}$ in diagonal (to ensure some overlap) seems the optimal FOV for a Digital Sunsensor.

The digital data-interface more or less automatically comes with the albedo insensitivity, but in order to keep the unit conductively cooled the interface will have to be selected to provide a low power dissipation.

To limit the power absorptions, the sensor must have a small Sun exposed area. This in turn will lead to the desired low weight as another contributor to the affordability of the solution. 